\title{
Fasting and meal-stimulated residual beta cell function is positively associated with serum concentrations of proinflammatory cytokines and negatively associated with anti-inflammatory and regulatory cytokines in patients with longer term type 1 diabetes
}

\author{
M. N. Pham • H. Kolb • T. Battelino • J. Ludvigsson • \\ P. Pozzilli • F. Zivehe • M. Roden • T. Mandrup-Poulsen • \\ N. C. Schloot • European C-Peptide Trial
}

Received: 18 October 2012 / Accepted: 14 February 2013 /Published online: 15 March 2013

(C) Springer-Verlag Berlin Heidelberg 2013

\begin{abstract}
Aims/hypothesis Cytokines may promote or inhibit disease progression in type 1 diabetes. We investigated whether systemic proinflammatory, anti-inflammatory and regulatory cytokines associated differently with fasting and meal-
\end{abstract}

stimulated beta cell function in patients with longer term type 1 diabetes.

Methods The beta cell function of 118 patients with type 1 diabetes of duration of $0.75-4.97$ years was tested using a standardised liquid mixed meal test (MMT). Serum samples

A complete list of investigators is available in the electronic supplementary material (ESM).

Electronic supplementary material The online version of this article (doi:10.1007/s00125-013-2883-3) contains peer-reviewed but unedited supplementary material, which is available to authorised users.

M. N. Pham $(\varangle) \cdot$ F. Zivehe $\cdot$ M. Roden $\cdot$ N. C. Schloot Institute for Clinical Diabetology at the German Diabetes Center, Leibniz Center for Diabetes Research at Heinrich-Heine-University Düsseldorf, Auf'm Hennekamp 65, 40225 Düsseldorf, Germany

e-mail: mnpham@gmx.de

H. Kolb

Institute of Molecular Medicine, Heinrich-Heine University,

Düsseldorf, Germany

T. Battelino

Department of Pediatric Endocrinology, Diabetes and Metabolism,

University Children's Hospital, Ljubljana, Slovenia

J. Ludvigsson

Division of Pediatrics, Department of Clinical and Experimental

Medicine, University of Linköping, Linköping, Sweden

\section{P. Pozzilli}

Department of Endocrinology and Diabetes, University Campus

BioMedico, Rome, Italy

P. Pozzilli

Centre of Diabetes, St Bartholomew's and the London, School of Medicine, Queen Mary, University of London, London, UK

M. Roden

Department of Metabolic Diseases, University Clinics Düsseldorf, Heinrich-Heine University, Düsseldorf, Germany

T. Mandrup-Poulsen

Department of Biomedical Sciences, Faculty of Health and Medical Sciences, University of Copenhagen, Copenhagen, Denmark

\section{T. Mandrup-Poulsen}

Department of Molecular Medicine and Surgery, Karolinska Institutet, Stockholm, Sweden 
obtained at -5 to 120 min were analysed by multiplex beadbased technology for proinflammatory (IL-6, TNF- $\alpha$ ), antiinflammatory (IL-1 receptor antagonist [IL-1RA]) and regulatory (IL-10, TGF- $\beta_{1-3}$ ) cytokines, and by standard procedures for C-peptide. Differences in beta cell function between patient groups were assessed using stepwise multiple regression analysis adjusting for sex, age, duration of diabetes, BMI, $\mathrm{HbA}_{1 \mathrm{c}}$ and fasting blood glucose.

Results High fasting systemic concentrations of the proinflammatory cytokines IL- 6 and TNF- $\alpha$ were associated with increased fasting and stimulated $\mathrm{C}$-peptide concentrations even after adjustment for confounders $(p<0.03)$. Interestingly, increased concentrations of anti-inflammatory/regulatory IL1RA, IL-10, TGF- $\beta_{1}$ and TGF- $\beta_{2}$ were associated with lower fasting and stimulated C-peptide levels $(p<0.04)$, losing significance on adjustment for anthropometric variables. During the MMT, circulating concentrations of IL- 6 and TNF- $\alpha$ increased $(p<0.001)$ while those of IL-10 and TGF- $\beta_{1}$ decreased $(p<0.02)$ and IL-1RA and TGF- $\beta_{2}$ remained unchanged.

Conclusions/interpretation The association between better preserved beta cell function in longer term type 1 diabetes and increased systemic proinflammatory cytokines and decreased anti-inflammatory and regulatory cytokines is suggestive of ongoing inflammatory disease activity that might be perpetuated by the remaining beta cells. These findings should be considered when designing immune intervention studies aimed at patients with longer term type 1 diabetes and residual beta cell function.

Keywords Beta cell function $\cdot$ C-peptide $\cdot$ Cytokine $\cdot$ Mixed meal $\cdot$ Type 1 diabetes

$\begin{array}{ll}\text { Abbreviations } \\ \text { CCL } & \text { Chemokine C-C motif ligand } \\ \text { ECPT } & \text { European C-Peptide Trial } \\ \text { IL-1RA } & \text { IL-1 receptor antagonist } \\ \text { MMT } & \text { Mixed meal test }\end{array}$

\section{Introduction}

Type 1 diabetes is an immune-mediated disease characterised by a destruction of pancreatic beta cells that is driven by islet reactive $\mathrm{T}$ cells $[1,2]$. Proinflammatory cytokines such as IL- $1 \beta$, IFN- $\gamma$ and TNF- $\alpha$ promote the destruction of pancreatic beta cells [3-5], while regulatory cytokines (IL-10 and TGF- $\beta_{1}$ ) [6, 7] and anti-inflammatory cytokines (IL-1 receptor antagonist [IL-1RA]) [8] can counteract beta cell destruction. An imbalance between pro- and anti-inflammatory and/or regulatory cytokines could be essential for the development of type 1 diabetes [9-11].

In the longitudinal Hvidøre Study of paediatric patients with newly diagnosed type 1 diabetes, an elevation of anti- inflammatory mediator (IL-1RA) and proinflammatory cytokine (IL-6) levels [12] was associated with remission and increased stimulated C-peptide, whereas IL-10, chemokine C-C motif ligand (CCL)2, CCL3, CCL4 and CCL5 concentrations [12-14] decreased over the first year after diagnosis of diabetes and were negatively associated with C-peptide level. A general immune activation at the time of diagnosis of diabetes may also result from increased expression of IFNG, TNF, IL6, IL4, TGFB and IL10 mRNA compared with patients with type 1 diabetes of more than 5 years' duration [15].

These findings of increased concentrations of pro- but also of anti-inflammatory/regulatory cytokines in patients with new-onset diabetes might reflect an attempt to counterregulate the ongoing beta cell destruction in patients with residual beta cell function. Based on these data, we hypothesised that patients with longer term type 1 diabetes with better preserved C-peptide function would also able to counteract the ongoing inflammatory process by an increased immune-regulatory status. Thus, we analysed the association between systemic anti-inflammatory, proinflammatory and regulatory cytokines, and fasting and mixed-meal-stimulated beta cell function in patients with type 1 diabetes within the first year of diagnosis and also in those with longer term diabetes with confirmed residual beta cell function. Furthermore, we investigated the influence of a high-protein liquid mixed meal test (MMT) on the systemic immune status of patients with type 1 diabetes, since the secretion of cytokines can be affected by food intake [16-19].

\section{Methods}

Study population According to the study protocol of the European C-Peptide Trial (ECPT), participants with type 1 diabetes and an age range of 8-40 years at the time of inclusion were allowed to participate the study. Type 1 diabetes was defined as insulin dependency from diagnosis onwards according to the WHO criteria. Participants receiving treatment with drugs influencing beta cell function and insulin sensitivity (e.g. steroids) and also significant concomitant disease likely to interfere with glucose metabolism (e.g. febrile illness) were excluded from the study. A total of 118 patients with type 1 diabetes and a duration of diabetes ranging from 0.75 to 4.97 years were included for the ECPT study (Table 1).

Patients underwent a standardised MMT by drinking a high-protein liquid formula (Boost HP; Mead Johnson Nutrition, Evansville, IN, USA) [20]. At -5, 0, 30, 90 and $120 \mathrm{~min}$, blood samples were drawn for metabolic and immunological analyses. Patients were fasted and had had no food or drink, and had not smoked, since 22:00 hours the 
Table 1 Clinical data for the study population

\begin{tabular}{ll}
\hline Patient characteristics & Value \\
\hline$n$ (female/male) & $118(48 / 70)$ \\
Age (years) & $19.4(8-40)$ \\
Duration of diabetes (years) & $2.3(0.75-4.97)$ \\
$\mathrm{HbA}_{1 \mathrm{c}}(\%)$ & $7.4(5.0-15.4)$ \\
$\mathrm{HbA}_{1 \mathrm{c}}(\mathrm{mmol} / \mathrm{mol})$ & $57.4(31.2-144.8)$ \\
$\mathrm{BMI}\left(\mathrm{kg} / \mathrm{m}^{2}\right)$ & $21.3(15.3-30.3)$ \\
Fasting glucose $(\mathrm{mmol} / \mathrm{l})$ & $8.7(3.6-22.8)$ \\
Baseline C-peptide $(\mathrm{pmol} / \mathrm{l})$ & $77(10.0-621.0)$ \\
\hline
\end{tabular}

Data are presented as median and range

previous day. Patients were advised against strenuous exercise on the morning of the test.

Table 1 shows the clinical characteristics of the patients. All the local ethics committees approved the study protocol, which was in accordance with the Declaration of Helsinki. All patients gave written informed consent to participate in the study.

Statistical methods To analyse our data, we used SAS Enterprise Guide Version 4.2 (SAS Institute, Cary, NC, USA) and GraphPad Prism version 4 for Windows (GraphPad Software, La Jolla, CA, USA).

A Kolmogorov-Smirnov test was carried out to test the normal distribution of the data. Comparisons of C-peptide concentrations between patient subgroups were made by multivariate regression analysis with stepwise adjustment for sex, age, duration of diabetes, $\mathrm{BMI}, \mathrm{HbA}_{1 \mathrm{c}}$ and fasting blood glucose. A Spearman correlation test was applied to analyse for a correlation between cytokine levels and metabolic variables. Comparisons of fasting circulating concentrations of immune mediators between patients with a duration of diabetes (1) <2 years; (2) 2-3 years; and (3) $>3$ years were carried out by a Kruskal-Wallis test followed by a post hoc (Dunn) test. Comparisons of circulating cytokine concentrations during the MMT were carried out using the Friedman test. The level of significance was set at $p<0.05$. Fasting data for the MMT were defined as the mean of the -5 and 0 min time points. Data on stimulated levels were defined as values of the 90 min time points during the MMT.

Biochemical measurements Serum samples were stored at $-20^{\circ} \mathrm{C}$ and thawed only once for measurement. We measured the proinflammatory cytokines IL- 6 and TNF- $\alpha$ and the regulatory cytokines IL- 10, TGF- $\beta_{1}$, TGF- $\beta_{2}$ and TGF- $\beta_{3}$ using multiplex bead-based technology in commercially available kits (Fluorokine MAP; R\&D Systems, Wiesbaden, Germany) [21-23]. To measure systemic concentrations of the anti-inflammatory cytokine IL-1RA, we used commercially available ELISA kits (Quantikine
ELISA kits; R\&D Systems). The detection limits of the assays were $0.04 \mathrm{pg} / \mathrm{ml}$ for IL- $6,0.24 \mathrm{pg} / \mathrm{ml}$ for TNF- $\alpha$, $0.02 \mathrm{pg} / \mathrm{ml}$ for IL-10, $37.8 \mathrm{pg} / \mathrm{ml}$ for IL-1RA, $10 \mathrm{pg} / \mathrm{ml}$ for TGF- $\beta_{1}, 5 \mathrm{pg} / \mathrm{ml}$ for TGF- $\beta_{2}$ and $10 \mathrm{pg} / \mathrm{ml}$ for TGF- $\beta_{3}$. For serum concentrations of immune mediators lower than the detection limit, a value half the detection limit was assigned, as previously reported $[12,21]$. The following proportions of values fell below the detection limit: $8 \%$ of IL- $6,6.8 \%$ of TNF- $\alpha, 0 \%$ of IL-1RA, $4.2 \%$ of IL- $10,0 \%$ of TGF- $\beta_{1}$ and TGF- $\beta_{2}$, and $60 \%$ of TGF- $\beta_{3}$ values. The results for TGF- $\beta_{3}$ were not considered in further analysis of the increased number of values falling below the detection limit. The cytokine assays showed an interassay variation of $<10 \%$ and an intra-assay variation of $<14 \%$.

The measurements of blood glucose, C-peptide and $\mathrm{HbA}_{1 \mathrm{c}}$ were performed at the central laboratory of the Steno Diabetes Center, Copenhagen, Denmark using fluoroimmunometric assay (AutoDELFIA; Perkin Elmer Wallac) as described elsewhere [20]. The interassay CV was $<6 \%[20]$.

\section{Results}

Association between beta cell function and systemic cytokine concentrations To assess the relationship between pancreatic beta cell function and systemic concentrations of proinflammatory, anti-inflammatory and regulatory cytokines, we divided patients with type 1 diabetes into two subgroups-low and high-depending on their median fasting immune mediator levels. Patients with lower than median systemic concentrations of fasting proinflammatory cytokines IL- 6 and TNF- $\alpha$ revealed a decreased level of fasting C-peptide (IL-6 $p=0.03$; TNF- $\alpha p=0.02$ ) compared with patients with higher fasting proinflammatory cytokines (Fig. 1). The same association was found when analysing for stimulated C-peptide (IL-6 $p=0.02$; TNF- $\alpha p=0.03$ ). These differences also remained significant in multiple regression analysis after stepwise adjustment for sex, age, BMI, duration of diabetes, $\mathrm{HbA}_{1 \mathrm{c}}$ and fasting blood glucose level.

For anti-inflammatory and regulatory cytokines, we found that patients with lower fasting concentrations of anti-inflammatory cytokine IL-1RA and regulatory cytokines IL-10, TGF- $\beta_{1}$ and TGF- $\beta_{2}$ had higher fasting C-peptide levels (IL-1RA $p=0.04$; IL-10 $p=0.03$; TGF- $\beta_{1}$ $p=0.04$; TGF- $\beta_{2} p=0.048$ ) (Fig. 1). For IL-1RA and IL-10, the same association was also found with stimulated C-peptide levels (IL-1RA $p=0.046$; IL-10 $p=0.04$ ). However, after using multivariate regression analysis with stepwise adjustments for sex, age, BMI, duration of diabetes, $\mathrm{HbA}_{1 \mathrm{c}}$ and fasting blood glucose level, we found that the significance of the association between fasting or 
Fig. 1 Comparisons of circulating C-peptide concentrations in patients with low and high concentrations of immune mediators. Patients were divided into subgroups below or above the median levels of fasting concentrations of immune mediators in order to investigate the association between immune mediator levels and fasting C-peptide concentrations. Regression analysis was used to assess $p$ values. Bars represent medians. $* p<0.05$
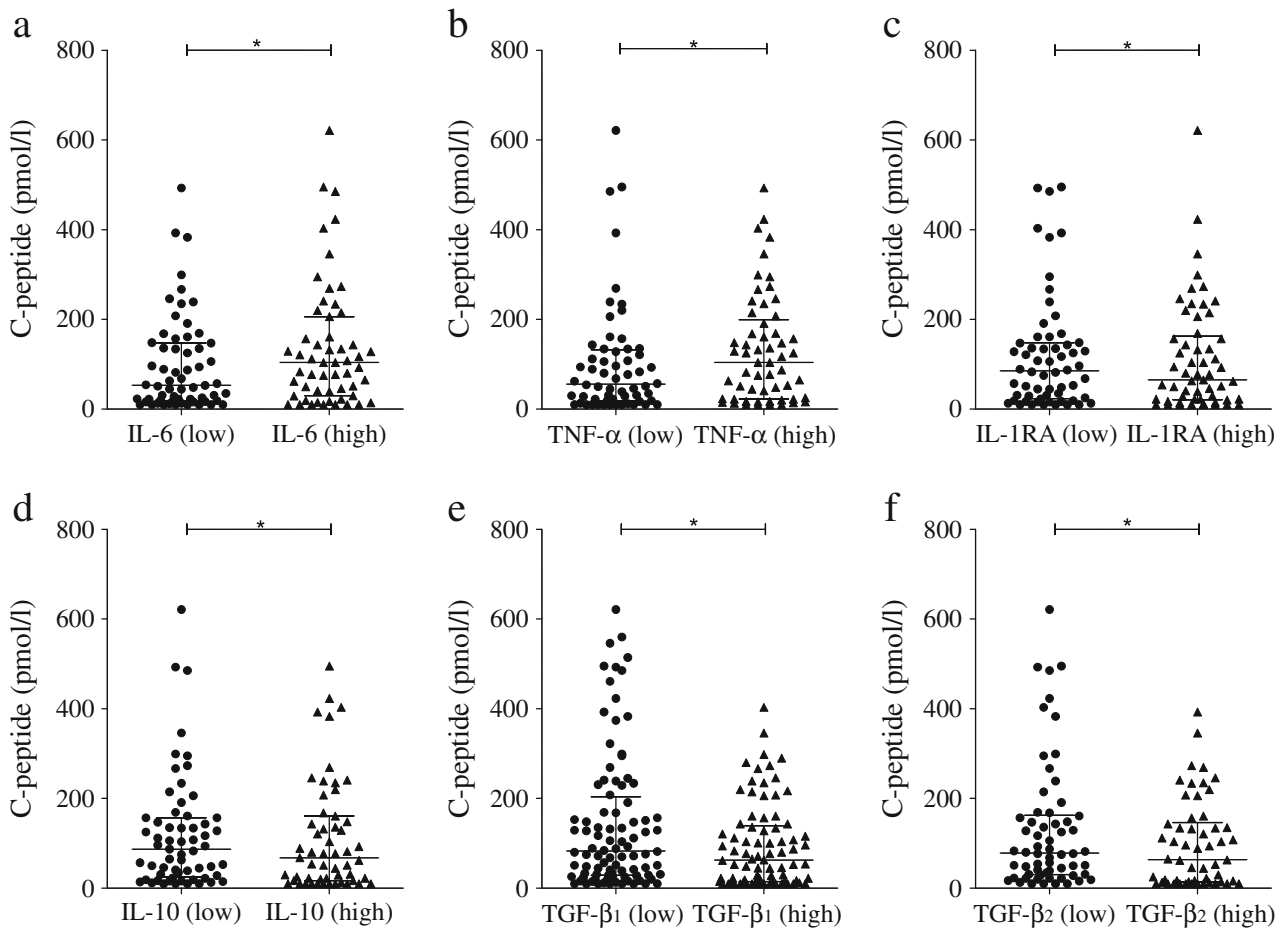

IL-1RA $(p=0.02)$ (Table 2). Systemic concentrations of fasting anti-inflammatory cytokine IL-1RA and regulatory cytokines IL-10 and TGF- $\beta_{2}$ showed a negative correlation with fasting as well as with stimulated beta cell function (all $p<0.05$ ), while fasting TGF- $\beta_{1}$ correlated negatively with fasting C-peptide $(p=0.04)$ (Table 2$)$. The regulatory cytokines IL-10, TGF- $\beta_{1}$ and TGF- $\beta_{2}$ correlated negatively with age (all $p<0.02$ ) (Table 2). Serum concentrations of TGF- $\beta_{2}$ correlated negatively with BMI and duration of diabetes (all $p<0.04$ ) (Table 2).

In our previous study [20,21], we found that the C-peptide levels decreased with a longer duration of diabetes, as expected. Based on these findings, we extended our analysis to the association between duration of diabetes and systemic concentrations of cytokines. We classified patients into three subgroups depending on their duration of diabetes (Fig. 2). We observed that patients with a duration of diabetes of less than 2 years had increased concentrations of proinflammatory

Table 2 Correlations between circulating concentrations of immune mediators and metabolic variables

\begin{tabular}{lllllll}
\hline Variable & BMI & Duration of diabetes & Age & HbA $_{1 \mathrm{c}}$ & Fasting C-peptide & Stimulated C-peptide \\
\hline IL-6 & $0.23(0.02)$ & $-0.23(0.01)$ & $0.19(0.05)$ & $0.001(0.99)$ & $0.20(0.002)$ & $0.11(0.03)$ \\
TNF- $\alpha$ & $0.29(0.002)$ & $-0.19(0.04)$ & $0.24(0.01)$ & $0.12(0.04)$ & $0.15(0.04)$ & $0.21(0.001)$ \\
IL 1RA & $0.08(0.40)$ & $-0.08(0.35)$ & $-0.04(0.66)$ & $-0.48(0.02)$ & $-0.16(0.03)$ & $-0.15(0.03)$ \\
IL-10 & $-0.11(0.27)$ & $0.11(0.23)$ & $-0.21(0.02)$ & $0.10(0.29)$ & $-0.14(0.05)$ & $-0.17(0.01)$ \\
TGF- $\beta_{1}$ & $-0.18(0.07)$ & $-0.13(0.20)$ & $-0.30(0.002)$ & $0.06(0.53)$ & $-0.19(0.04)$ & $-0.07(0.06)$ \\
TGF- $\beta_{2}$ & $-0.20(0.04)$ & $-0.24(0.01)$ & $-0.35(0.0002)$ & $0.06(0.52)$ & $-0.18(0.02)$ & $-0.14(0.01)$ \\
\hline
\end{tabular}

Correlation analyses were carried out by Spearman test. Data are presented as Spearman correlation coefficients $r$ with $p$ values in brackets 
Fig. 2 Comparisons of circulating concentrations of immune mediators between patient groups with different durations of diabetes. Patients were classified into three subgroups depending on their duration of diabetes. The Kruskal-Wallis test followed by a post hoc (Dunn) test was used to assess $p$ values. Bars represent medians. ${ }^{*} p<0.05$, $* * p<0.01$
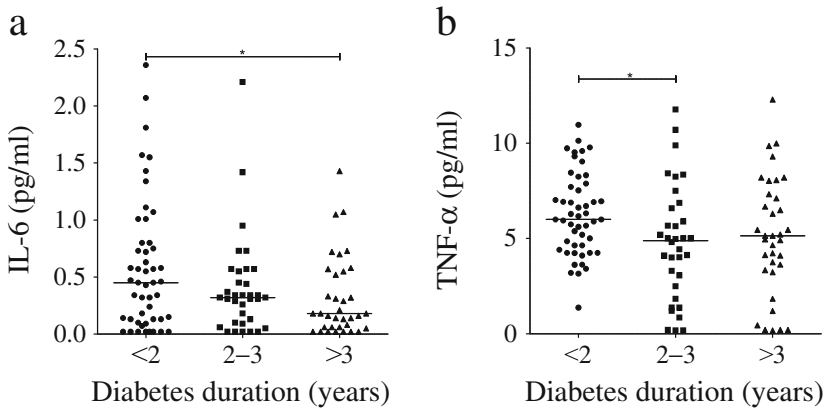

Diabetes duration (years)

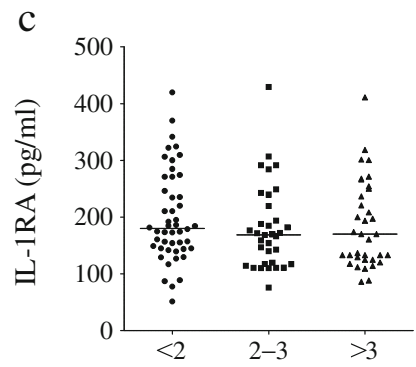

Diabetes duration (years)

e
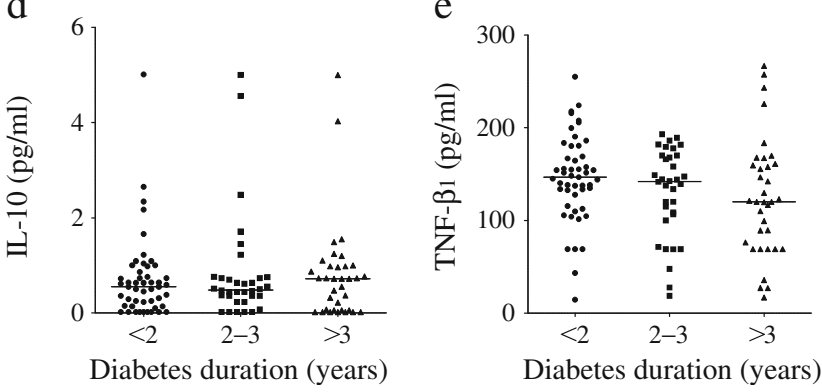

f

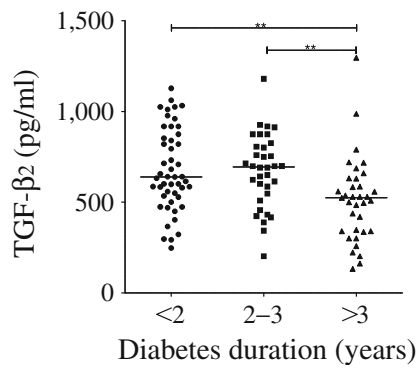

cytokines IL- 6 and TNF- $\alpha$ compared with those with a duration of diabetes from $2-3$ years (TNF- $\alpha p=0.03$ ) and more than 3 years (IL-6 $p=0.04$ ), while the levels of antiinflammatory cytokine IL-1RA and regulatory cytokines IL10 and TGF- $\beta_{1}$ were similar between all subgroups (Fig. 2). Patient subgroups with a duration of diabetes of less than 2 years and $2-3$ years (all $p=0.001$ ) had higher concentrations of TGF- $\beta_{2}$ than those who had been diagnosed for more than 3 years (Fig. 2). Taking the data together, residual C-peptide concentrations showed an association with systemic immune status dominated by increased proinflammatory cytokines in patients with higher C-peptide levels.

In an additional analysis, we performed a Spearman correlation test to investigate the possible association

between proinflammatory, anti-inflammatory and regulatory cytokines. The systemic concentrations of proinflammatory cytokines IL- 6 and TNF- $\alpha$ did not show any correlations with each other or with anti-inflammatory cytokine IL-1RA and regulatory cytokines IL- 10, TGF- $\beta_{1}$ and TGF- $\beta_{2}$. However, we observed that the concentrations of IL-1RA correlated positively with TGF- $\beta_{1}(r=0.34, p=0.0003)$ and TGF- $\beta_{2}(r=0.28, p=0.0004)$, and TGF- $\beta_{1}$ correlated positively with TGF- $\beta_{2}(r=0.70, p<0.0001)$. Thereby, antiinflammatory/regulatory cytokines were associated with each other but not with proinflammatory cytokines.

Systemic concentrations of cytokines during the liquid MMT During the MMT, the levels of C-peptide were

Fig. 3 Systemic concentrations of immune mediators in patients with type 1 diabetes during the liquid MMT. Median and interquartile range (Q1-Q3) are shown. The Friedman test followed by a post hoc test (Dunn's test) was used to assess $p$ values. ${ }^{*} p<0.05,{ }^{* * *} p<0.001$
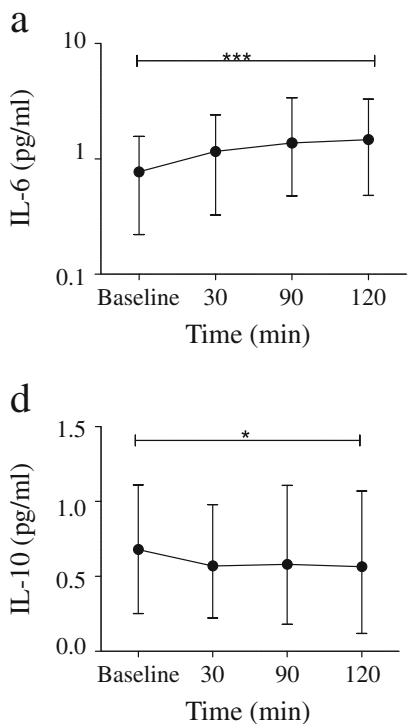

b
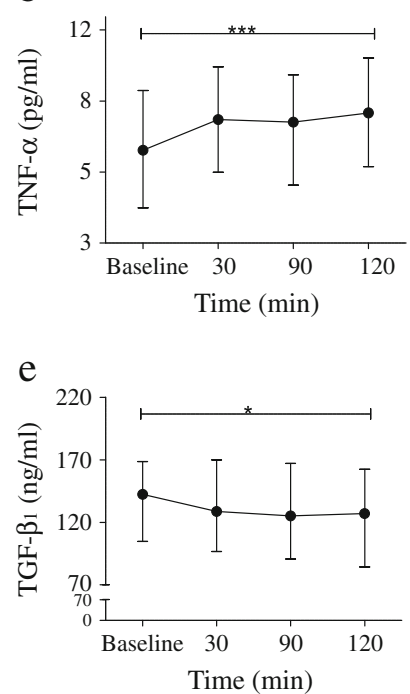
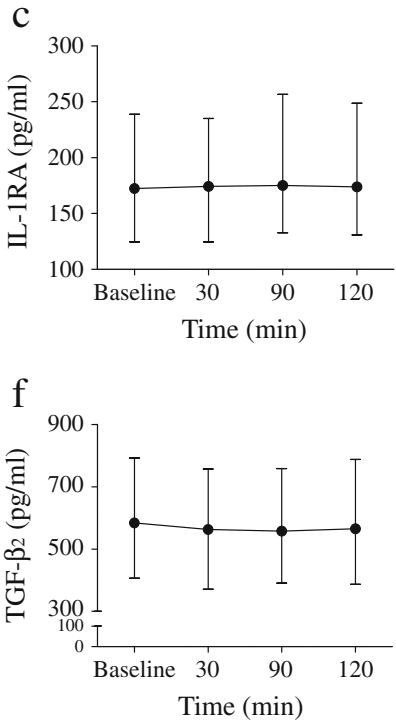
increased, as expected [20]. Circulating concentrations of the proinflammatory cytokines IL-6 $(p<0.0001)$ and TNF- $\alpha$ $(p=0.001)$ increased significantly, whereas IL-1RA and TGF- $\beta_{2}$ values remained stable and IL-10 $(p=0.02)$ and TGF- $\beta_{1}(p=0.01)$ decreased during the MMT (Fig. 3). The increase from fasting to $120 \mathrm{~min}$ for IL-6 was $91 \%$ and for TNF- $\alpha$ was $27 \%(p<0.0001)$ (Fig. 3). The patient subgroup with increased (above median) fasting cytokines showed an increase of $75 \%$ for IL- 6 and $25 \%$ for TNF- $\alpha$ over $120 \mathrm{~min}$ (all $p<0.0001$ ). The decrease from fasting to $120 \mathrm{~min}$ was $18 \%$ for IL-10 $(p=0.02)$ and $10 \%$ for TGF- $\beta_{1}(p=0.04)$ (Fig. 3). The patient subgroup above the fasting median tended to show a decrease in level of $9 \%$ for IL-10 and $2 \%$ for TGF- $\beta_{1}$.

\section{Discussion}

We found that fasting systemic concentrations of proinflammatory cytokines TNF- $\alpha$ and IL- 6 in patients with type 1 diabetes were positively associated with fasting and stimulated C-peptide even after adjustment for metabolic and anthropometric variables. Fasting systemic concentrations of the anti-inflammatory cytokine IL-1RA and regulatory cytokines IL-10, TGF- $\beta_{1}$ and TGF- $\beta_{2}$ showed a negative association with pancreatic beta cell function, but this relationship was lost after adjustment for anthropometric measures.

The opposite association with residual endogenous C-peptide secretion for cytokines with opposing function suggests a biological relevance. A higher secretion capacity for C-peptide probably indicates a larger residual beta cell mass. It is conceivable that the presence of beta cells promotes beta cell-directed immune reactivity and inflammation. Indeed, histological post mortem analyses of pancreases from patients with type 1 diabetes have demonstrated local inflammation of the islets only if beta cells were still present [24]. The insulitis process is thought to decrease during the years after onset of diabetes, in parallel with the loss of residual beta cell function. We found a decrease in systemic concentrations of the proinflammatory cytokines TNF- $\alpha$ and IL- 6 with a longer duration of diabetes.

Both cytokines are known to associate with obesity and may be released from obese adipose tissue [25]. We confirm the positive association of BMI with IL- 6 and TNF- $\alpha$ in our group of patients with type 1 diabetes. However, adjustment for BMI did not abolish the positive association between C-peptide and systemic IL- 6 and TNF- $\alpha$ concentration. Therefore, we conclude that systemic IL- 6 and TNF- $\alpha$ probably reflect ongoing islet inflammation. It has been shown in NOD mice and rat models of insulin-dependent diabetes mellitus that pancreatic cytokine levels correlate with systemic cytokine concentrations and the degree of insulitis or islet destruction [26, 27]. Interestingly, findings in mice show that IL- 6 can enhance insulin secretion by increasing glucagon-like peptide-1 secretion from L-cells and alpha-cells [28].

It is interesting that we did not detect a correlation between individual serum concentrations of IL- 6 and TNF- $\alpha$. There was also no correlation between the two cytokines during the more acute disease stage 1 month after diagnosis of type 1 diabetes [12]. The two proinflammatory cytokines may therefore reflect different qualities of islet inflammation and/or may be released from different sites in the body.

There was no negative correlation between the two proinflammatory cytokines and IL-1RA, IL-10 or TGF- $\beta_{1-3}$. This suggests a lack of interdependence, which fits with the observation that concentrations of the antiinflammatory/regulatory cytokines were not associated with residual beta cell function. In contrast, we previously found a possible protective effect of IL-1RA during the first year after diagnosis of diabetes, i.e. a positive association between IL-1RA levels and stimulated C-peptide concentrations [12]. Peak expression of anti-inflammatory counterregulatory cytokines may occur prior to the onset of overt diabetes, as seen in the siblings of patients with type 1 diabetes or in animal models [23, 27], whereas the diseaserelated production of these cytokines may regress several years after diagnosis of diabetes, as observed here. Previous studies have reported that these anti-inflammatory and regulatory cytokines are able to protect pancreatic beta cells from immune destruction [8, 29-31]. The lack of such activity may promote the persistence of the beta cell destructive process in patients with a longer duration of diabetes.

During the MMT, we observed an increase in proinflammatory cytokines (IL-6 and TNF- $\alpha$ ) and a decrease in regulatory mediators (IL-10 and TGF- $\beta_{1}$ ). An acute meal-induced increase in IL- 6 and TNF- $\alpha$ is known from previous studies with patients with the metabolic syndrome and type 2 diabetes and healthy overweight controls, but has not yet been determined in patients with type 1 diabetes [16-19]. Furthermore, no postprandial data are available for anti-inflammatory mediators, i.e. IL-1RA, IL10 , TGF- $\beta_{1}$ or TGF- $\beta_{2}$. The data from the mixed meal intake in our study show that the acute food intake of a high-protein drink slightly increases proinflammatory cytokine concentrations and decreases anti-inflammatory and regulatory cytokines. It is not known whether mealinduced systemic cytokines affect islet function or whether cytokine production is elicited directly in the islet tissue. Therefore, the effect of post-meal systemic immune activation on beta cell survival remains to be elucidated.

As we cannot conclude from our observations that the islets are the source of cytokine production and do not know the mechanism behind the effects of these altered cytokine 
levels upon a mixed meal intake, these observations require further mechanistic study.

The current study has some limitations. Although patients with recognisable acute infections were not included in the study, there may have been cases of subclinical infectious disease. Second, in line with the ECPT study protocol, patients had had no food or drink (with the exception of water) and had not smoked since 22:00 hours the preceding day, and were advised against strenuous exercise on the morning of the MMT. However, as smoking and strenuous exercise are difficult to control in outpatient conditions, this is a limitation of the study as smoking and exercise might have influenced the outcome of the MMT. Third, this study is cross-sectional and the investigated data related to immune mediators in these patients may not apply to individual clinical situations owing to the high variability of cytokine levels between individuals. Longitudinal studies of changes in cytokine levels over time may possibly provide information with relevance for individual patients.

In conclusion, the association between better preserved beta cell function in longer term type 1 diabetes and increased systemic proinflammatory cytokines and decreased anti-inflammatory and regulatory cytokines is suggestive of ongoing inflammatory disease activity that might be perpetuated by the remaining beta cells and could potentially be influenced by nutrient composition. These findings should be considered when designing immune intervention studies aimed at patients with longer term type 1 diabetes and residual beta cell function.

Acknowledgements This study was performed with samples from the European C-Peptide Trial (ECPT) Study Group. We thank all clinical investigators, study nurses and patients. N. C. Schloot is a guest scientist at the German Diabetes Center, Düsseldorf, Germany and currently employed by Lilly Deutschland GmbH, Bad Homburg, Germany.

Funding The ECPT was supported by the European Foundation for the Study of Diabetes (EFSD). This project was supported in part by the German Diabetes Foundation (DDG) and Deutsches Zentrum für Diabetesforschung (DZD) e.V.

Duality of interest The authors declare that there is no duality of interest associated with this manuscript.

Contribution statement MNP researched, interpreted and analysed the data, and wrote and revised the manuscript. NCS participated in the clinical ECPT study, acquired and interpreted the data, and wrote and revised the manuscript. TM-P and HK are both in positions of authority within the ECPT and initiated it. HK contributed to conception, design, analysis and interpretation of the data, and to the discussion, writing and revision of the manuscript. TM-P acquired, researched and interpreted the data, and contributed to the discussion, interpretation and revision of the manuscript. FZ acquired and revised the data. TB, JL, PP and MR analysed and interpreted the data and revised the article for important intellectual content. All authors approved the final version.

\section{References}

1. Bettini M, Vignali DA (2011) T cell-driven initiation and propagation of autoimmune diabetes. Curr Opin Immunol 23:754-760

2. Varanasi V, Avanesyan L, Schumann DM, Chervonsky AV (2012) Cytotoxic mechanisms employed by mouse $\mathrm{T}$ cells to destroy pancreatic $\beta$-cells. Diabetes 61:2862-2870

3. Rabinovitch A, Suarez-Pinzon WL (1998) Cytokines and their roles in pancreatic islet beta-cell destruction and insulindependent diabetes mellitus. Biochem Pharmacol 55:1139-1149

4. Donath MY, Størling J, Berchtold LA, Billestrup N, MandrupPoulsen T (2008) Cytokines and beta-cell biology: from concept to clinical translation. Endocr Rev 29:334-350

5. Mandrup-Poulsen T, Pickersgill LMS, Donath MY (2010) Blockade of interleukin 1 in type 1 diabetes mellitus. Nat Rev Endocrinol 6:158-166

6. Wållberg M, Wong FS, Green EA (2011) An islet-specific pulse of TGF- $\beta$ abrogates CTL function and promotes $\beta$ cell survival independent of Foxp3+ T cells. J Immunol 186:2543-2551

7. Tai N, Yasuda H, Xiang Y et al (2011) IL-10-conditioned dendritic cells prevent autoimmune diabetes in NOD and humanized HLADQ8/RIP-B7.1 mice. Clin Immunol 139:336-349

8. Ardestani A, Sauter NS, Paroni F et al (2011) Neutralizing interleukin-1beta (IL-1beta) induces beta-cell survival by maintaining PDX1 protein nuclear localization. J Biol Chem 286:17144-17155

9. Rachmiel M, Bloch O, Bistritzer T et al (2006) TH1/TH2 cytokine balance in patients with both type 1 diabetes mellitus and asthma. Cytokine 34:170-176

10. Christen U, von Herrath MG (2004) Manipulating the type 1 vs type 2 balance in type 1 diabetes. Immunol Res 30:309-325

11. Karlsson MG, Lawesson SS, Ludvigsson J (2000) Th1-like dominance in high-risk first-degree relatives of type I diabetic patients. Diabetologia 43:742-749

12. Pfleger C, Mortensen HB, Hansen L et al (2008) Association of IL$1 \mathrm{ra}$ and adiponectin with C-peptide and remission in patients with type 1 diabetes. Diabetes 57:929-937

13. Pfleger C, Kaas A, Hansen L et al (2008) Relation of circulating concentrations of chemokine receptor CCR5 ligands to C-peptide, proinsulin and $\mathrm{HbA}_{1 \mathrm{c}}$ and disease progression in type 1 diabetes. Clin Immunol 128:57-65

14. Kaas A, Pfleger C, Kharagjitsingh AV et al (2012) Association between age, IL-10, IFN $\gamma$, stimulated C-peptide and disease progression in children with newly diagnosed type 1 diabetes. Diabet Med 29:734-741

15. Han D, Leyva CA, Matheson D et al (2011) Immune profiling by multiple gene expression analysis in patients at-risk and with type 1 diabetes. Clin Immunol 139:290-301

16. Peluso I, Raguzzini A, Villano DV et al (2012) High fat meal increase of IL-17 is prevented by ingestion of fruit juice drink in healthy overweight subjects. Curr Pharm Des 18:85-90

17. Poppitt SD, Keogh GF, Lithander FE et al (2008) Postprandial response of adiponectin, interleukin-6, tumor necrosis factor-alpha, and C-reactive protein to a high-fat dietary load. Nutrition 24:32232-32239

18. Nappo F, Esposito K, Cioffi M et al (2002) Postprandial endothelial activation in healthy subjects and in type 2 diabetic patients: role of fat and carbohydrate meals. J Am Coll Cardiol 39:11451150

19. Esposito K, Ciotola M, Sasso FC et al (2007) Effect of a single high-fat meal on endothelial function in patients with the metabolic syndrome: role of tumor necrosis factor-alpha. Nutr Metab Cardiovasc Dis 17:274-279

20. Greenbaum CJ, Mandrup-Poulsen T, McGee PF et al (2008) Mixed-meal tolerance test versus glucagon stimulation test for 
the assessment of beta-cell function in therapeutic trials in type 1 diabetes. Diabetes Care 31:1966-1971

21. Pham MN, Hawa MI, Pfleger C et al (2011) Pro- and antiinflammatory cytokines in latent autoimmune diabetes in adults, type 1 and type 2 diabetes patients: Action LADA 4. Diabetologia 54:1630-1638

22. Highfill SL, Kelly RM, O'Shaughnessy MJ et al (2009) Multipotent adult progenitor cells can suppress graft-versus-host disease via prostaglandin E2 synthesis and only if localized to sites of allopriming. Blood 114:693-701

23. Olivieri A, De Angelis S, Dionisi S et al (2010) Serum transforming growth factor $\beta 1$ during diabetes development in non-obese diabetic mice and humans. Clin Exp Immunol 162:407-414

24. Foulis AK, Farquharson MA (1986) Aberrant expression of HLADR antigens by insulin-containing beta-cells in recent-onset type I diabetes mellitus. Diabetes 35:1215-1224

25. Shoelson SE, Herrero L, Naaz A (2007) Obesity, inflammation, and insulin resistance. Gastroenterology 132:2169-2180
26. Jörns A, Rath KJ, Terbish T et al (2010) Diabetes prevention by immunomodulatory FTY720 treatment in the LEW.1AR1-iddm rat despite immune cell activation. Endocrinology 151:3555-3565

27. Schloot NC, Hanifi-Moghaddam P, Goebel C et al (2002) Serum IFN-gamma and IL-10 levels are associated with disease progression in non-obese diabetic mice. Diabetes Metabol Res Rev 18:64-70

28. Ellingsgaard H, Hauselmann I, Schuler B et al (2011) Interleukin-6 enhances insulin secretion by increasing glucagon-like peptide-1 secretion from L cells and alpha cells. Nat Med 17:1481-1489

29. Parsa R, Andresen P, Gillett A et al (2012) Adoptive transfer of immunomodulatory M2 macrophages prevents type 1 diabetes in NOD mice. Diabetes 61:2881-2892

30. Subramanian L, Blumenfeld H, Tohn R et al (2012) NKT cells stimulated by long fatty acyl chain sulfatides significantly reduces the incidence of type 1 diabetes in nonobese diabetic mice. PLoS One 7:e37771

31. Yi Z, Diz R, Martin AJ et al (2012) Long-term remission of diabetes in NOD mice is induced by nondepleting anti-CD4 and anti-CD8 antibodies. Diabetes 61:2871-2880 